\title{
Erratum
}

\section{Soft-breaking correction to hard supersymmetric relations}

\section{QCD correction to squark decay}

Ken-ichi Hikasa, Yumi Nakamura

Department of Physics, Tohoku University, Aoba-ku, Sendai 980-77, Japan

Z. Phys. C 70 (1996) 139-143

The sign of the diagram with a gluino is in error. Equation (18) should read

$$
\begin{aligned}
& F_{\dot{g}}=\sqrt{R r}\left\{\frac{R+r-2}{(1-r)^{2}} H(R, r)+\frac{1}{r} \log (1-r)\right. \\
&\left.+\frac{1}{1-r}[R \log R-(R-1) \log |R-1|]\right\}
\end{aligned}
$$

Equation (22) should read

$$
\begin{aligned}
F= & -2 \operatorname{Li}_{2}(r)-\log r \log (1-r)-\frac{\pi^{2}}{3}-\frac{3}{2} \log (1-r) \\
& -\frac{r(4-3 r)}{4(1-r)^{2}} \log r+\frac{13-14 r}{4(1-r)}+\frac{3}{4} \log R-1 \\
& -\frac{1}{2}\left[\left(R^{2}-1\right) \log \frac{|R-1|}{R}+R\right] \\
+ & \frac{1}{4}\left[\frac{2 R-1}{(R-1)^{2}} \log R-\frac{1}{R-1}\right] \\
+ & \sqrt{R r}\left\{\frac{R+r-2}{(1-r)^{2}} H(R, r)+\frac{1}{r} \log (1-r)\right. \\
& \left.+\frac{1}{1-r}[R \log R-(R-1) \log |R-1|]\right\} .
\end{aligned}
$$

This error affects Figs. 4 and 5. Conclusions of the paper remain unchanged. We are grateful to $P$. Zerwas for correspondence; the DESY-Leiden group found the error independently.

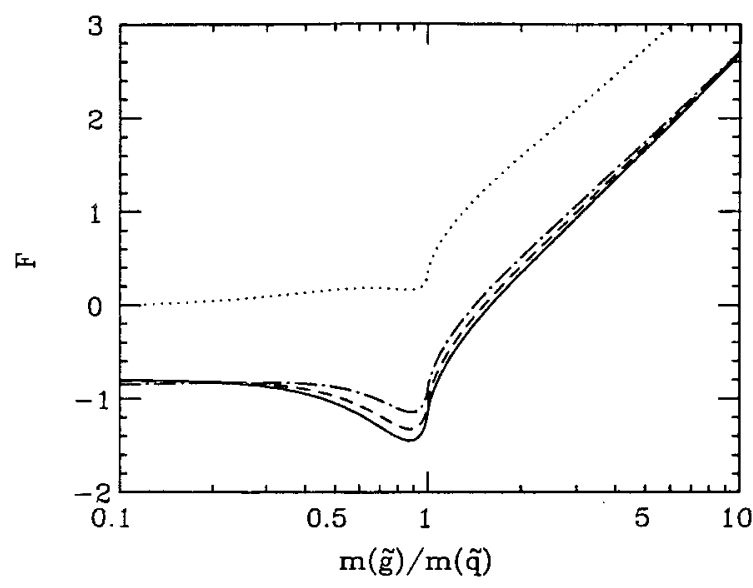

Fig. 4

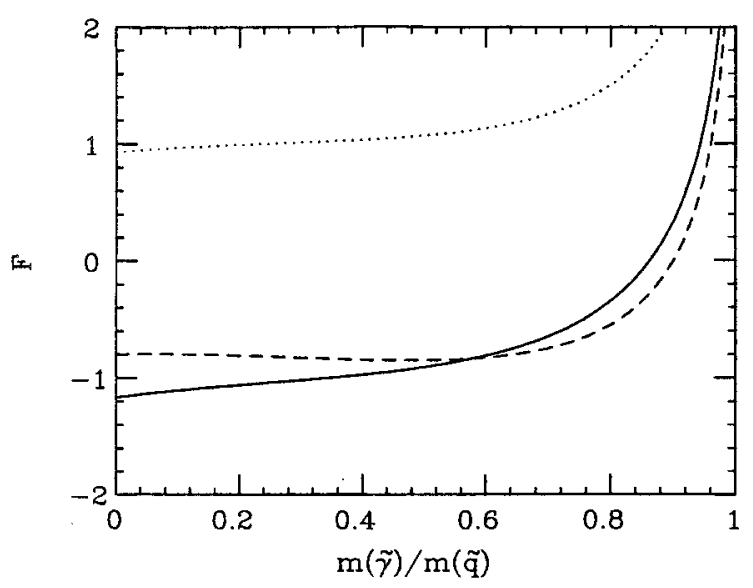

Fig. 5 